\title{
Emergency Conversion to General Anesthesia Is a Tolerable Risk in Patients Undergoing Mechanical Thrombectomy
}

\author{
(D) F. Flottmann, (D) Leischner, (D) G. Broocks, (D)T.D. Faizy, (D)A. Aigner, (D) M. Deb-Chatterji, (D) G. Thomalla, (D). Krauel,
} (D) M. Issleib, (D). Fiehler, and (D) C. Brekenfeld

\begin{abstract}
BACKGROUND AND PURPOSE: Mechanical thrombectomy for acute ischemic stroke is performed with the patient under local anesthesia, conscious sedation, or general anesthesia. According to recent trials, up to $16 \%$ of patients require emergency conversion to general anesthesia during mechanical thrombectomy. This study investigated the procedural and clinical outcomes after emergency conversion in comparison with local anesthesia, conscious sedation, and general anesthesia.
\end{abstract}

MATERIALS AND METHODS: This retrospective study included 254 patients undergoing mechanical thrombectomy for acute largevessel occlusion. The procedure was started with the patient either under local anesthesia, conscious sedation, or general anesthesia. Emergency conversion was defined as induction of general anesthesia during mechanical thrombectomy. The primary outcomes were successful reperfusion ( $\mathrm{TICl} 2 \mathrm{~b} / 3$ ) and functional independence (mRS at 90 days, $\leq 2$ ).

RESULTS: Twenty-five patients (9.8\%) required emergency conversion to general anesthesia. The time from admission to flow restoration was increased under general anesthesia (median, 137 minutes) and emergency conversion (median, 138 minutes) compared with local anesthesia (median 110 minutes). After adjustment for confounders, emergency conversion to general anesthesia and primary general anesthesia had comparable chances of successful reperfusion $(O R=1.28 ; 95 \% \mathrm{Cl}, 0.31-5.25)$. Patients with emergency conversion had a tendency toward higher chances of functional independence $(\mathrm{OR}=4.48 ; 95 \% \mathrm{Cl}, 0.49-40.86)$ compared with primary general anesthesia, but not compared with local anesthesia $(\mathrm{OR}=0.86 ; 95 \% \mathrm{Cl}, 0.14-5.11)$ and conscious sedation $(\mathrm{OR}=1.07 ; 95 \% \mathrm{Cl}, 0.17-6.53)$.

CONCLUSIONS: Patients with emergency conversion did not have lower chances of successful reperfusion or functional independence compared those with primary general anesthesia, and time to flow restoration was also similar. We found no evidence supporting the primary induction of general anesthesia in patients at risk for emergency conversion.

ABBREVIATIONS: $\mathrm{CS}=$ conscious sedation; $\mathrm{GA}=$ general anesthesia; $\mathrm{MAP}=$ mean arterial pressure

$\mathrm{n}$ acute ischemic stroke due to large-vessel occlusion, mechanical thrombectomy combined with standard treatment has been shown to be more effective than standard therapy alone and is now considered standard of care. ${ }^{1-6}$ However, the optimal anesthesia management during thrombectomy is still controversial. ${ }^{7-10}$

Patients are usually allocated to either local anesthesia, conscious sedation (CS), or general anesthesia (GA) before initiation of mechanical thrombectomy. However, up to $16 \%$ of patients

Received May 18, 2019; accepted after revision October 1.

From the Department of Diagnostic and Interventional Neuroradiology (F.F., H.L., G.B., T.D.F., J.F., C.B.), Institute of Medical Biometry and Epidemiology (A.A.), and Departments of Neurology (M.D.-C., G.T.) and Anaesthesiology (J.K., M.I.),

University Medical Center Hamburg-Eppendorf, Hamburg, Germany; and Institute of Public Health (A.A.), Charité-Universitätsmedizin Berlin, Berlin, Germany.

Please address correspondence to Fabian Flottmann, MD, Department of

Diagnostic and Interventional Neuroradiology, University Medical Center

Hamburg-Eppendorf, Haus O22, Martinistr 52, 20246 Hamburg, Germany;

e-mail: f.flottmann@uke.de

Indicates article with supplemental on-line table.

http://dx.doi.org/10.3174/ajnr.A6321 who undergo thrombectomy under initial local anesthesia or CS require emergency conversion to GA during the procedure, often due to severe agitation or loss of airway-protective reflexes. ${ }^{11-13}$ The impact of conversion to GA on procedural and clinical outcomes is unknown.

We therefore conducted a retrospective study with the aim of comparing procedural and clinical outcomes of patients requiring emergency conversion to GA with those of patients undergoing treatment under either local anesthesia, CS, or GA for the entire intervention. Our hypotheses were that there are differences in the time from admission to flow restoration and in the numbers of successful reperfusions and/or good clinical outcomes between emergency conversion and the 3 groups with other anesthesia management.

\section{MATERIALS AND METHODS \\ Patient Selection}

Between June 2015 and January 2018, all patients older than 18 years of age who underwent endovascular treatment for acute 
ischemic stroke of the anterior circulation at the University Medical Center Hamburg-Eppendorf were enrolled. During this time, local anesthesia with or without CS was the preferred anesthesia management. GA was induced before mechanical thrombectomy only if deemed necessary due to clinical conditions (coma, apnea, loss of airway protection, uncontrollable patient movement) or if patients arrived already intubated following transfer from another hospital.

Study procedures were conducted following the protocol of the German Stroke Registry in accordance with ethical guidelines (Ethics Committee of the Hamburg Chamber of Physicians, Hamburg, Germany) and in compliance with the Declaration of Helsinki. Informed consent was obtained from all capable patients or available legal representatives.

\section{Intervention and Airway Management}

All patients were under the supervision of an anesthesiologist during the entire procedure. Mechanical thrombectomy was initiated with the patient under either local anesthesia, CS, or GA. Local anesthesia or CS was the preferred protocol in nonagitated, compliant patients with patent airways. The decision to induce GA during mechanical thrombectomy was made by both the neurointerventionalist and the anesthesiologist in cases of excessive patient movement, complications, respiratory failure, or loss of airway-protective reflexes.

During CS and GA, the final combination and dosages of the administered anesthetics were left to the discretion of the attending anesthesiologist. Propofol was the preferred sedative, either fractionized or continuously administered $(1-3 \mathrm{mg} / \mathrm{kg} / \mathrm{h})$. Remifentanil, midazolam, and piritramide were used if propofol was deemed inappropriate or insufficient.

GA was induced following preoxygenation with an opioid for systemic anesthesia, a bolus of propofol for sedation, and rocuronium or suxamethonium for relaxation as a rapid sequence induction. Airway patency was maintained by endotracheal intubation and mechanical ventilation. Anesthesia was maintained with propofol (2-10 $\mathrm{mg} / \mathrm{kg} / \mathrm{h})$ and an opioid.

Throughout the procedure, the patient's oxygenation, ventilation, and hemodynamics were continually monitored. Care was taken to avoid hypotension to maintain sufficient perfusion of penumbral tissue ${ }^{14}$ by the perfusor-controlled infusion of norepinephrine (titrated in steps of $0.05-0.1 \mu \mathrm{g} / \mathrm{kg} / \mathrm{min}$ ). After emergency conversion or GA, all patients were transferred to a neurointensive care unit without prior extubation in the neurointerventional suite.

\section{Data Acquisition and Analysis}

Baseline data were documented, as well as prehospital mRS and admission NIHSS. Procedural data included time intervals between symptom onset (if available), admission, imaging, groin puncture and reperfusion, and complications during and after mechanical thrombectomy. Anesthesiology protocols were reviewed for the type of anesthesia management, administered medication, and blood pressure records. A drop of blood pressure was defined as a $>25 \%$ decrease of mean arterial blood pressure (MAP) during the intervention before achieving intracranial reperfusion. Clinical outcome parameters included NIHSS and mRS scores at 24 hours and at discharge and the mRS score at 90 days. Functional independence was defined as mRS at 90 days $\leq 2$.

\section{Image Analysis}

All image analyses were performed by an experienced neuroradiologist ( $>5$ years of experience). Baseline ASPECTS was routinely assessed on preintervention nonenhanced CT scans or diffusionweighted images. ${ }^{15}$ The collateral score was determined on admission CT angiography using the system of Maas et al. ${ }^{16}$ TICI scores were determined in a final angiographic series, and TICI $2 b / 3$ was considered indicative of successful reperfusion, which was the primary procedural outcome.

\section{Statistical Analysis}

Categoric variables are reported as absolute and relative frequencies; continuous variables, as mean and SD when approximate normal distribution holds, and as median and first and third quartiles (Q1, Q3) otherwise. To better compare the median time from admission to flow restoration among groups, we estimated the $95 \%$ bootstrap confidence intervals of the differences in medians. ${ }^{17,18}$ We applied logistic regression to model the associations between the primary procedural and clinical outcomes and the 4 anesthesia groups (local anesthesia, CS, emergency conversion, GA), adjusting for relevant confounders: age, sex, admission NIHSS, prestroke mRS, comorbidities (systolic blood pressure on admission, arterial hypertension, diabetes, dyslipidemia, atrial fibrillation, active smoking), application of intravenous thrombolysis, location of vessel occlusion, and, additionally, for successful reperfusion and occurrence of a significant drop in blood pressure in the analysis of functional independence. On the basis of these models, we performed a likelihood-ratio $\chi^{2}$ test to assess global differences in outcomes among the 4 groups as implemented in the $\mathrm{R}$ car package ${ }^{19}$ and derived OR estimates along with 95\% CIs. Individual comparisons among the anesthesia groups were adjusted with the Tukey method, based on the $\mathrm{R}$ multcomp package ${ }^{20}$ visualized with the ggplot2 package. ${ }^{21}$

As a subset analysis, we performed the same models, additionally adjusting for collateral status and the time from symptom onset to reperfusion due to a high number of missing values in these variables. Instead of 238 observations with complete datasets, models were then based on 110 observations. Analyses were performed with the statistical software $\mathrm{R}^{22}$

\section{RESULTS}

\section{Baseline Characteristics}

Of the 307 patients treated with mechanical thrombectomy in the designated time period, 255 patients met the inclusion criteria. One patient had to be excluded due to missing anesthesia records. Of the 254 included patients, $53 \%$ were women, and the mean age was $72.1 \pm 12.6$ years. The median NIHSS score on admission was $15(\mathrm{Q} 1-\mathrm{Q} 3,12-19)$, and $15 \%$ of patients presented with a prestroke $m R S$ of $\geq 2$. The median ASPECTS on admission imaging was 7 (range, 6-9). GA was induced before endovascular treatment in 59 patients (23.2\%). In 94 patients (37.0\%), endovascular treatment was completed with the patient under local anesthesia, 76 patients (29.9\%) had CS, and 25 patients (9.8\%) required emergency conversion during the procedure (Table 1). 


\begin{tabular}{|c|c|c|c|c|}
\hline & $\begin{array}{l}\text { Local Anesthesia Only } \\
\qquad(n=94)\end{array}$ & CS $(n=76)$ & $\begin{array}{c}\text { Emergency Conversion to } \\
\text { GA }(n=25)\end{array}$ & GA $(n=59)$ \\
\hline Age (mean) (SD) (yr) & $74.3(11.2)$ & $71.7(12.4)$ & $67.8(13.9)$ & $71.0(14.1)$ \\
\hline Female sex (No.) (\%) & 55 (58.5\%) & $36(47.4 \%)$ & $12(48.0 \%)$ & $31(52.5 \%)$ \\
\hline Hypertension (No.) (\%) & $66(70.2 \%)$ & $54(71.1 \%)$ & $15(60.0 \%)$ & $37(62.7 \%)$ \\
\hline Diabetes mellitus (No.) (\%) & 15 (16.0\%) & $17(22.4 \%)$ & $4(16.0 \%)$ & $9(15.3 \%)$ \\
\hline Dyslipidemia (No.) (\%) & $11(11.7 \%)$ & $13(17.1 \%)$ & $2(8.0 \%)$ & $11(18.6 \%)$ \\
\hline Atrial fibrillation (No.) (\%) & $42(44.7 \%)$ & $27(35.5 \%)$ & $7(28.0 \%)$ & 24 (40.7\%) \\
\hline Active smoker (No.) (\%) & $11(11.7 \%)$ & $10(13.2 \%)$ & $2(8.0 \%)$ & $10(16.9 \%)$ \\
\hline $\begin{array}{l}\text { Systolic blood pressure at admission } \\
\text { (mean) (SD) (mm Hg) }\end{array}$ & $154(28.0)$ & $162(27.9)$ & $166(22.8)$ & $156(33.9)$ \\
\hline Pre-Stroke $\mathrm{mRS} \geq 2$ & 14 (14.9\%) & $12(15.8 \%)$ & $2(8.0 \%)$ & $11(18.6 \%)$ \\
\hline Initial NIHSS (median) (Q1-Q3) & $15.0(11-18)$ & $15.0(11.0-18.0)$ & $17.0(13.0-19.0)$ & $18.0(15.0-20.5)$ \\
\hline Initial ASPECTS (median) (Q1-Q3) & $8(7-9)$ & $8(6-8)$ & $7(5-9)$ & $7(6-8)$ \\
\hline Collaterals on CTA ${ }^{a}$ & $2(1-3)$ & $2(1-3)$ & $2(1-3)$ & $2(1-2)$ \\
\hline \multicolumn{5}{|l|}{ Initial occlusion site (No.) (\%) } \\
\hline Left hemisphere & $130(51.2 \%)$ & $46(48.9 \%)$ & $39(51.3 \%)$ & $13(52.0 \%)$ \\
\hline Tandem occlusion & $11(11.7 \%)$ & $14(18.4 \%)$ & $3(12.0 \%)$ & $9(15.3 \%)$ \\
\hline ICA (non-terminal) & $6(6.38 \%)$ & $4(5.26 \%)$ & $1(4.00 \%)$ & $3(5.08 \%)$ \\
\hline ICA (terminal segment) & $9(9.57 \%)$ & $18(23.7 \%)$ & $3(12.0 \%)$ & $15(25.4 \%)$ \\
\hline M1 proximal & $32(34.0 \%)$ & 19 (25.0\%) & $13(52.0 \%)$ & $22(37.3 \%)$ \\
\hline M1 distal & $21(22.3 \%)$ & $13(17.1 \%)$ & $2(8.00 \%)$ & $7(11.9 \%)$ \\
\hline M2 & $15(16.0 \%)$ & $8(10.5 \%)$ & $3(12.0 \%)$ & $3(5.08 \%)$ \\
\hline Intravenous tPA (No.) (\%) & 57 (60.6\%) & $46(60.5 \%)$ & $17(68.0 \%)$ & $32(54.2 \%)$ \\
\hline $\begin{array}{l}\text { Onset to admission (median) (Q1-Q3) } \\
\left(\text { (min) }^{\mathrm{b}}\right.\end{array}$ & $176(115-226)$ & $193(124-236)$ & $131(65.0-176)$ & 181 (139-239) \\
\hline
\end{tabular}

available for 185 patients.

${ }^{\mathrm{b}}$ Available for 153 patients.

Patients with emergency conversion had a higher median NIHSS at admission compared with patients treated under local anesthesia and CS, but a lower median NIHSS than patients treated under GA (emergency conversion: 17 versus local anesthesia and CS, 15, versus GA, 18). The median initial ASPECTS on imaging was lower in patients with emergency conversion and GA (emergency conversion and GA, 7, versus local anesthesia and CS, 8). Proximal M1 occlusions were more frequent in patients with emergency conversion compared with patients with GA (emergency conversion, $52.0 \%$, versus GA, $37.3 \%$ ).

\section{Reasons for Emergency Conversion, Anesthesia Management, and Procedural Data}

Before emergency conversion, 17/25 patients (68\%) received sedative agents. Sedative agents used before emergency conversion were propofol $(n=10)$, remifentanil $(n=3)$, propofol and piritramide $(n=2)$, and midazolam $(n=2)$.

The median time from groin puncture to emergency conversion was 35 minutes (Q1-Q3, 25-60 minutes). The median number of passes before conversion to GA was 0 (Q1-Q3, 0-1). Reported reasons for emergency conversion were excessive patient movement $(n=18)$, neurologic deterioration $(n=4)$, periprocedural hemorrhage $(n=1)$, intention of stent placement in the intracranial ICA $(n=1)$, and emesis $(n=1)$.

None of the patients had a complication as a result of emergency conversion.

\section{Time Intervals from Admission to Flow Restoration}

Patients with emergency conversion had a shorter median time from admission to imaging than patients with primary GA (emergency conversion, 13.5 minutes, versus GA, 18 minutes) and from imaging to groin puncture (emergency conversion, 50.5 minutes, versus GA, 77.5 minutes). However, in patients who required emergency conversion during mechanical thrombectomy, the time from groin puncture to flow restoration was longer compared with local anesthesia, CS, and GA (emergency conversion, 79 minutes, versus GA, 51.5 minutes; local anesthesia, 39 minutes; CS, 47 minutes).

Overall, the time from admission to flow restoration was longer in patients with emergency conversion compared with those receiving local anesthesia (emergency conversion [median], 138 minutes, versus local anesthesia, 110 minutes; difference of medians, 28 minutes; 95\% CI, 4.5-54.5 minutes) but not longer than in those undergoing GA (emergency conversion, 138 minutes, versus GA, 137 minutes; difference of medians, 1 minute; 95\% CI, -22-35.5 minutes).

\section{Proportion of Successful Reperfusion}

Overall, successful reperfusion (TICI 2b/3) was achieved in $69.1 \%$ of all patients, but proportions varied among the 4 groups (local anesthesia, 79.8\%; CS, 69.7\%, emergency conversion, 64.0\%; GA, $54.2 \%$; Table 2).

On the basis of the multivariable logistic regression, the chances of successful reperfusion differed significantly among the 4 anesthesia groups $(P$ value $=.002)$. However, there were no significant differences between emergency conversion and the other 3 groups, especially not between emergency conversion and GA (Fig 1; OR $=1.28 ; 95 \%$ CI, 0.31-5.25). There was a tendency toward lower chances of successful reperfusion after emergency conversion compared with local anesthesia $(\mathrm{OR}=0.26$; 95\% CI, $0.06-1.06)$ and, to a lesser extent, compared with $\mathrm{CS}(\mathrm{OR}=0.55$; 95\% CI, 0.13-2.18). 


\begin{tabular}{|c|c|c|c|c|}
\hline & Local Anesthesia & CS & Emergency Conversion to GA & GA \\
\hline MR imaging before intervention (No.) (\%) & $20(21.3 \%)$ & $10(13.2 \%)$ & $3(12.0 \%)$ & $4(6.78 \%)$ \\
\hline Time from admission to imaging (median) (Q1-Q3) & $16.0(10.8-23.2)$ & $13.5(8-22.2)$ & $13.5(9.75-20.2)$ & $18.0(13.0-34.0)$ \\
\hline $\begin{array}{l}\text { Time from imaging to groin puncture (median) } \\
\text { (Q1-Q3) }\end{array}$ & $49.5(40.0-65.5)$ & $51.0(41.0-73.2)$ & $50.5(37.0-61.5)$ & $77.5(56.5-94.2)$ \\
\hline $\begin{array}{l}\text { Time from groin puncture to flow restoration } \\
\text { (median) (Q1-Q3) }\end{array}$ & $39.0(24.8-61.2)$ & $47.0(30.0-70.2)$ & $79.0(60.0-110)$ & $51.5(30.0-69.0)$ \\
\hline $\begin{array}{l}\text { Time from admission to flow restoration (median) } \\
\text { (Q1-Q3) }\end{array}$ & $110(87.2-133)$ & $118(94.8-140)$ & 138 (116-179) & $137(114-177)$ \\
\hline \multicolumn{5}{|l|}{ Mechanical thrombectomy technique (No.) (\%) } \\
\hline Stent retriever only & $41(43.6 \%)$ & $40(52.6 \%)$ & $16(64.0 \%)$ & $29(49.2 \%)$ \\
\hline Aspiration only & $18(19.1 \%)$ & $7(9.21 \%)$ & $3(12.0 \%)$ & $8(13.6 \%)$ \\
\hline Both Stent retriever and aspiration & $21(22.3 \%)$ & $24(31.6 \%)$ & $6(24.0 \%)$ & $15(25.4 \%)$ \\
\hline $\begin{array}{l}\text { No endovascular treatment attempt } \\
\text { (spontaneous reperfusion or failure of access) }\end{array}$ & $14(14.9 \%)$ & $5(6.58 \%)$ & $0(0 \%)$ & $7(11.9 \%)$ \\
\hline Successful reperfusion (TICl 2b/3) (No.) (\%) & $75(79.8 \%)$ & $53(69.7 \%)$ & $16(64.0 \%)$ & $32(54.2 \%)$ \\
\hline \multicolumn{5}{|l|}{ Periprocedural complications (No.) (\%) } \\
\hline Dissections & $5(5.32 \%)$ & $1(1.32 \%)$ & $1(4.00 \%)$ & $1(1.69 \%)$ \\
\hline Hypotonia ( $>25 \%$ drop of MAP) & $2(2.13 \%)$ & $4(5.26 \%)$ & $7(28.0 \%)$ & $7(11.9 \%)$ \\
\hline
\end{tabular}

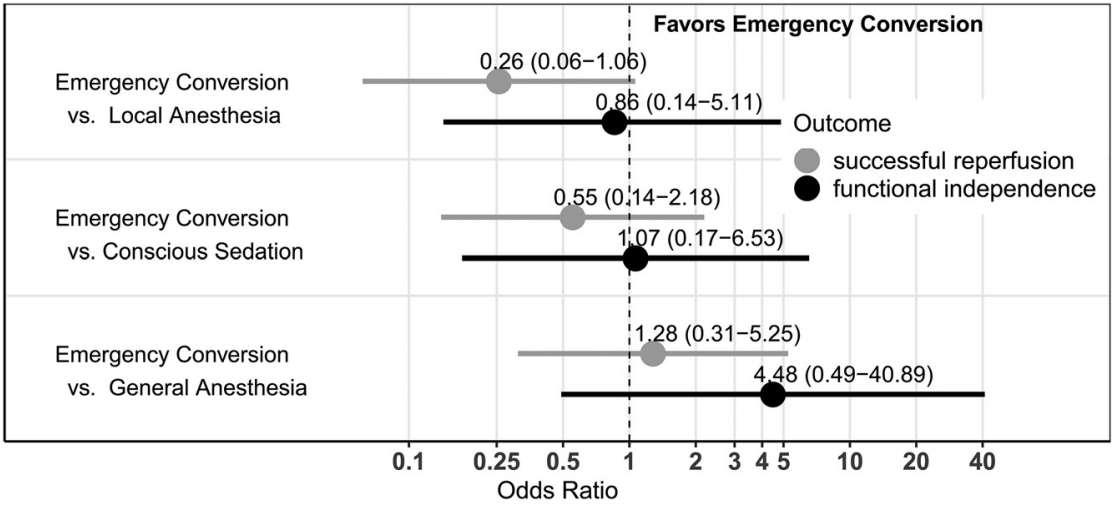

FIG 1. Adjusted odds ratio estimates with $95 \%$ confidence intervals comparing patients after emergency conversion with those who received local anesthesia, conscious sedation, and primary general anesthesia for successful reperfusion and functional independence. Successful reperfusion is $\mathrm{TICl} 2 \mathrm{~b} / 3$ on the final angiogram, $P=.002$, for global differences across all groups (likelihood-ratio $\chi^{2}$ test). Functional independence was mRS at 90 days $\leq 2, P=.12$, for global differences across all groups (likelihood-ratio $\chi^{2}$ test). Results are derived from logistic regression models, adjusted for confounders (age, sex, admission NIHSS, prestroke mRS $>1$, ASPECTS on admission imaging, comorbidities, application of IV thrombolysis, location of vessel occlusion and, additionally, TICI $2 b / 3$ and occurrence of a drop of MAP in the analysis of functional independence) and based on 238 complete observations.

In the subset analysis extending the model with time from symptom onset and collateral score, there was no longer any significant difference among the 4 anesthesia groups $(P$ value $=.52)$. When we compared emergency conversion and GA in this subset analysis, emergency conversion showed a tendency toward lower odds of successful reperfusion (On-line Table; OR $=0.26 ; 95 \%$ CI, 0.01-5.04).

\section{Clinical Outcomes}

At 24 hours, at discharge, and at 90 days, patients after local anesthesia and CS had the lowest NIHSS and mRS scores, while emergency conversion and GA scores were comparable (Table 3). The proportion of functional independence was highest after local anesthesia (32.2\%), followed by CS (29.2\%), emergency conversion (28.0\%), and GA (7.4\%).
In the multivariable logistic regression, differences of functional independence among the groups were not statistically significant $(P$ value $=.12)$. In our cohort, patients with emergency conversion had higher odds of good outcome compared with those under GA (OR $=4.48$; 95\% CI, 0.4940.89), but this effect was not statistically significant. Patients with emergency conversion had outcomes similar to those with local anesthesia (OR= 0.86; 95\% CI, 0.14-5.1) and CS (OR= 1.07; 95\% CI, 0.17-6.53).

In the subset analysis with time from symptom onset and collateral score, there was again no significant difference among the anesthesia groups $(P$ value $=$ $.15)$. Again, emergency conversion had higher chances of functional independence than $\mathrm{GA}(\mathrm{OR}=14.34 ; 95 \% \mathrm{CI}$, $0.14-1448.78)$.

Blood Pressure during Intervention

In patients who underwent emergency conversion, an unwanted drop of blood pressure $>25 \%$ of initial MAP occurred in $28.0 \%$ of patients, followed by GA (11.9\%), CS (5.3\%), and local anesthesia $(2.1 \%)$. However, the occurrence of a drop of MAP was not significantly associated with functional independence in multivariable logistic regression $(\mathrm{OR}=1.32$; 95\% CI, 0.29-5.78).

\section{DISCUSSION}

During mechanical thrombectomy, patients are treated under either local anesthesia, CS, or GA. In this retrospective study, 1 in 10 patients required emergency conversion to GA at some point during the intervention. The main finding of this study is that emergency conversion did not delay the time from admission to 


\begin{tabular}{lcccc}
\hline & Local Anesthesia & CS & Emergency Conversion to GA & GA \\
\hline Symptomatic intracranial hemorrhage (No.) (\%) & $3(3.19 \%)$ & $5(6.58 \%)$ & $1(4.00 \%)$ & $3(5.08 \%)$ \\
NIHSS at 24 hr (median) (Q1-Q3) & $9(4-16.8)$ & $12.5(6-20.0)$ & $19.0(14.5-42.0)$ & $21.0(15.0-42.0)$ \\
Change in NIHSS at 24 hr (median) (Q1-Q3) & $-3(-9.75-0.00)$ & $-1(-8.00-4.00)$ & $0(-2.50-21.5)$ & $3(-1.00-9.50)$ \\
mRS at 24 hr (median) (Q1-Q3) & $4(3-5)$ & $5(3-5)$ & $5(5-5)$ & $5(5-5)$ \\
NIHSS at discharge (median) (Q1-Q3) & $6(2-11.0)$ & $6(2-13.0)$ & $10.5(6.50-18.2)$ & $13.0(10.0-18.0)$ \\
mRS at discharge (median) (Q1-Q3) & $4(2-5)$ & $4(2-5)$ & $5(3-5)$ & $5(4-5)$ \\
mRS at 90 days (median) (Q1-Q3) & $4(1.50-5)$ & $4(2-6)$ & $5(2-5)$ & $5(4-6)$ \\
mRS 0-2 at 90 days (No.) (\%) & $28(32.2 \%)$ & $21(29.2 \%)$ & $7(28.0 \%)$ & $4(7.41 \%)$ \\
\hline
\end{tabular}

${ }^{a}$ Data were available for $87 / 94$ patients (local anesthesia),72/76 patients (CS), 25/25 patients (emergency conversion), and 54/59 patients (GA).

reperfusion compared with primary GA, and emergency conversion did not cause deterioration of clinical outcome.

Both emergency conversion and primary GA resulted in a similar delay in the time from admission to flow restoration of 19-20 minutes compared with CS, and 27-28 minutes compared with local anesthesia. This result is in accordance with the times for primary GA induction reported in recently published randomized trials, which ranged from 10 to 27 minutes. ${ }^{1-13}$ Emergency conversion is performed under suboptimal conditions in comparison with primary GA (intubation on the angiography table versus the premedication room). However, emergency conversion was not associated with a loss of time compared with primary GA. Furthermore, no complications were reported as a result of emergency conversion to GA.

In approximately three-quarters of all patients, thrombectomy was initiated with the patient under local anesthesia or CS. Of these, $13 \%$ required emergency conversion. This number is comparable with the results of the SIESTA trial $(14.3 \%, 11$ of 77 patients under $\mathrm{CS}$ converted to $\mathrm{GA})^{11}$ and the AnStroke trial (15.6\%, 7/45 patients), ${ }^{12}$ while the GOLIATH trial reported a smaller proportion of emergency conversion (6.3\%, 4/63 patients). ${ }^{13}$ Emergency conversion seems to be a common phenomenon during mechanical thrombectomy. In our study, we could not identify a clear pattern of anesthesia medication preceding emergency conversion to GA. Approximately one-third of patients received only local anesthesia without sedation, one-third received propofol only, and the remaining third was sedated with either benzodiazepines or opioids.

The main reason reported for emergency conversion during thrombectomy was excessive patient movement/agitation $(72.0 \%$, $18 / 25$ patients), while 4 patients exhibited neurologic deterioration. In the SIESTA trial, the main reason for emergency conversion was also severe agitation (63.6\%, 7/11 patients). ${ }^{11}$ The AnStroke trial reported puncture of the common carotid artery as the main reason for conversion to GA (57.1\%, 4/7 patients), while only 2 patients were intubated due to severe agitation. ${ }^{12}$

A drop of MAP during mechanical thrombectomy has been linked to unfavorable outcome. ${ }^{23}$ We found a higher proportion of an unwanted drop of MAP in patients under emergency conversion (28\%), followed by GA (12\%). However, in the multivariable analysis conducted for the present study, the occurrence of an unwanted drop of MAP was not significantly associated with an unfavorable outcome.

With regard to clinical outcome after emergency conversion, there are 2 scenarios: First, one could hypothesize that emergency conversion is especially harmful compared with primary GA due to the suboptimal conditions of GA induction (intubation in the angiography suite, less time for preparation, more frequent occurrence of a drop of blood pressure, and so forth.). In this case, all patients with even the slightest risk of emergency conversion, for example due to excessive movement on admission, should be intubated beforehand. Second, if emergency conversion is not especially harmful, one could initially perform local anesthesia/CS, even in these at-risk patients. To address this point, we compared all types of anesthesia in multivariable analysis for functional independence. Generally, we did not find any statistically significant differences between emergency conversion and the other types of anesthesia. We found a tendency toward higher chances of good clinical outcome after emergency conversion compared with primary GA, albeit it was not statistically significant. Therefore, CS-policy treatment centers should not hesitate to initiate treatment under local anesthesia/CS, even in patients at risk of emergency conversion.

The overall rate of successful perfusion in our patient cohort was similar to that in the HERMES meta-analysis of 5 randomized controlled trials ( $71 \%$ versus $69 \%$ in our patient cohort). ${ }^{24}$ In our multivariable analysis, emergency conversion was not associated with decreased rates of successful reperfusion compared with GA. There was a tendency toward lower chances of successful reperfusion after emergency conversion compared with local anesthesia and CS. In the literature, the type of anesthesia seems to have little influence on the rate of successful reperfusion, as has been reported for CS and GA, both in a large meta-analysis of retrospective studies and in recent prospective trials. ${ }^{8,11-13}$

In this single-center study, local anesthesia with or without CS was the method of choice. Primary GA was induced before thrombectomy only if necessary. Thus, patients with severe strokes more frequently received primary GA, as has been previously shown. ${ }^{25-27}$ Observational studies on the type of anesthesia in mechanical thrombectomy are subject to selection bias because primary GA is typically induced in more severely affected patients. ${ }^{8}$ Indeed, in this study, patients with emergency conversion fell between patients with CS and GA in terms of stroke severity and possibly had selection bias as well. However, we controlled for both symptom severity, prestroke mRS, successful reperfusion, and location of occlusion in the multivariable analysis and additionally for time from symptom onset to reperfusion and collateral score in a subset analysis.

Compared with larger randomized trials, patients in our study showed lower rates of functional independence: The HERMES meta-analysis of 5 randomized controlled trials ${ }^{24}$ reported functional independence in $46 \%$ of patients in the intervention group 
versus only $25 \%$ of patients in our cohort. This result is most likely because compared with the HERMES cohort, patients in our study were older (72 versus 68 years) and had lower ASPECTS at admission (median, 9 versus 7). Both factors were associated with poor outcome in the HERMES meta-analysis. Furthermore, a substantive proportion of our patients presented with prestroke $m R S \geq 2$ (15\%).

This study reflects cases from a single institution. Results could differ for emerging technical approaches of mechanical thrombectomy. ${ }^{28}$ Furthermore, as in other retrospective studies on the type of anesthesia used in mechanical thrombectomy, selection bias is possible. The number of patients in the emergency conversion group was comparatively small, resulting in large confidence intervals, especially in the subset analysis, and results may differ for larger and/or multicenter patient cohorts. In addition, the reasons for induction of primary GA were not reported. The decision to administer sedation in patients with CS or to convert a patient under CS to GA was based on clinical indications and made individually by the attending neurointerventionalist and/or anesthesiologist.

\section{CONCLUSIONS}

In comparison with primary GA, emergency conversion to GA did not result in prolonged time to flow restoration, and chances of successful reperfusion and functional independence were not decreased. We found no evidence supporting the primary induction of GA in patients at risk for emergency conversion.

Disclosures: Annette Aigner-UNRELATED: Employment: University Medical Center Hamburg, Charité-Universitätsmedizin Berlin; Payment for Lectures Including Service on Speakers Bureaus: Hamburg University of Applied Sciences, Bernhard-Nocht Institute of Tropical Medicine, Roche Diagnostics, University of Salzburg, Charité-Universitätsmedizin Berlin, Studienstiftung des Deutschen Volkes, Hamburger Fern-Hochschule, Universität Heidelberg. Götz ThomallaUNRELATED: Consultancy: Acandis, Stryker; Payment for Lectures Including Service on Speakers Bureaus: Bayer, Bristol Myers Squibb, Boehringer Ingelheim, Daiichi Sankyo. Jens Fiehler-UNRELATED: Consultancy: Acandis, Boehringer Ingelheim, Cerenovus, Covidien, Evasc Neurovascular, MD-Clinicals, Medtronic, Medina, MicroVention, Penumbra, Route 92 Medical, Stryker, Transverse Medical; Grants/Grants Pending: MicroVention, Medtronic, Stryker, Cerenovus.* Malte Issleib-UNRELATED: Employment: UKE-Center for Anasthesiology and Intensive Care. *Money paid to the institution.

\section{ACKNOWLEDGMENT}

We would like to thank Rosalie McDonough for reviewing the manuscript.

\section{REFERENCES}

1. Berkhemer OA, Fransen PS, Beumer D, et al. A randomized trial of intraarterial treatment for acute ischemic stroke. $N$ Engl $\mathrm{J} \mathrm{Med}$ 2015;372:11-20 CrossRef Medline

2. Saver JL, Goyal M, Bonafe A, et al. Stent-retriever thrombectomy after intravenous t-PA vs. t-PA alone in stroke. $N$ Engl $J$ Med 2015;372:2285-95 CrossRef Medline

3. Campbell BC, Mitchell PJ, Kleinig TJ, et al. Endovascular therapy for ischemic stroke with perfusion-imaging selection. $N$ Engl J Med 2015;372:1009-18 CrossRef Medline

4. Jovin TG, Chamorro A, Cobo E, et al. Thrombectomy within 8 hours after symptom onset in ischemic stroke. $N$ Engl J Med 2015;372:2296-2306 CrossRef Medline
5. Goyal M, Demchuk AM, Menon BK, et al. Randomized assessment of rapid endovascular treatment of ischemic stroke. $N$ Engl J Med 2015;372:1019-30 CrossRef Medline

6. Bhogal P, Andersson T, Maus V, et al. Mechanical thrombectomy-a brief review of a revolutionary new treatment for thromboembolic stroke. Clin Neuroradiol 2018;28:313-26 CrossRef Medline

7. Brinjikji W, Murad MH, Rabinstein AA, et al. Conscious sedation versus general anesthesia during endovascular acute ischemic stroke treatment: a systematic review and meta-analysis. AJNR Am J Neuroradiol 2015;36:525-29 CrossRef Medline

8. Brinjikji W, Pasternak J, Murad MH, et al. Anesthesia-related outcomes for endovascular stroke revascularization. Stroke 2017;48: 2784-91 CrossRef Medline

9. Ilyas A, Chen CJ, Ding D, et al. Endovascular mechanical thrombectomy for acute ischemic stroke under general anesthesia versus conscious sedation: a systematic review and meta-analysis. World Neurosurg 2018;12:e355-67 CrossRef Medline

10. Campbell BV, van Zwam WH, Goyal M, et al. Effect of general anaesthesia on functional outcome in patients with anterior circulation ischaemic stroke having endovascular thrombectomy versus standard care: a meta-analysis of individual patient data. Lancet Neurol 2018;17:47-53 CrossRef Medline

11. Schönenberger S, Uhlmann L, Hacke W, et al. Effect of conscious sedation vs general anesthesia on early neurological improvement among patients with ischemic stroke undergoing endovascular thrombectomy. JAMA 2016;316:1986 CrossRef Medline

12. Löwhagen Hendén P, Rentzos A, Karlsson JE, et al. General anesthesia versus conscious sedation for endovascular treatment of acute ischemic stroke. Stroke 2017;48:1601-07 CrossRef Medline

13. Simonsen CZ, Yoo AJ, Sørensen LH, et al. Effect of general anesthesia and conscious sedation during endovascular therapy on infarct growth and clinical outcomes in acute ischemic stroke. JAMA Neurol 2018;75:470-77 CrossRef Medline

14. Brekenfeld C, Mattle HP, Schroth G. General is better than local anesthesia during endovascular procedures. Stroke 2010;41:2716-17 CrossRef Medline

15. Barber PA, Demchuk AM, Zhang J, et al. Validity and reliability of a quantitative computed tomography score in predicting outcome of hyperacute stroke before thrombolytic therapy: ASPECTS Study Group-Alberta Stroke Programme Early CT Score. Lancet 2000;355:1670-74 CrossRef Medline

16. Maas MB, Lev MH, Ay $\mathrm{H}$, et al. Collateral vessels on CT angiography predict outcome in acute ischemic stroke. Stroke 2009;40:300105 CrossRef Medline

17. Canty A, Ripley BD. Bootstrap R (S-Plus) Functions. 2019. https:// astrostatistics.psu.edu/su07/R/html/boot/html/00Index.html. Accessed ????????

18. Davison AC, Hinkley DV. Bootstrap Methods and Their Applications. Cambridge: Cambridge University Press; 1997

19. Fox J, Weisberg S. An R Companion to Applied Regression. Thousand Oaks: Sage Publication; 2011

20. Hothorn T, Bretz F, Westfall P. Simultaneous inference in general parametric models. Biom J 2008;50:346-63 CrossRef Medline

21. Wickham H. Ggplot2 Elegant Graphics for Data Analysis. New York: Springer-Verlag; 2016

22. R Core Team. R: A Language and Environment for Statistical Computing. https://cran.r-project.org/doc/manuals/fullrefman.pdf. Accessed ?????

23. Treurniet KM, Berkhemer OA, Immink RV, et al. A decrease in blood pressure is associated with unfavorable outcome in patients undergoing thrombectomy under general anesthesia. J Neurointerv Surg 2018;10:107-11 CrossRef Medline

24. Goyal M, Menon BK, van Zwam WH, et al; HERMES collaborators. Endovascular thrombectomy after large-vessel ischaemic stroke: a meta-analysis of individual patient data from five randomised trials. Lancet 2016;387:1723-31 CrossRef Medline 
25. Abou-Chebl A, Yeatts SD, Yan B, et al. Impact of general anesthesia on safety and outcomes in the endovascular arm of Interventional Management of Stroke (IMS) III Trial. Stroke 2015;46:2142-48 CrossRef Medline

26. van den Berg LA, Koelman DH, Berkhemer OA, et al. Type of anesthesia and differences in clinical outcome after intra-arterial treatment for ischemic stroke. Stroke 2015;46:1257-62 CrossRef Medline
27. Berkhemer OA, van den Berg LA, Fransen PSS, et al. The effect of anesthetic management during intra-arterial therapy for acute stroke in MR CLEAN. Neurology 2016;87:656-64 CrossRef Medline

28. Maegerlein C, Berndt MT, Mönch S, et al. Further development of combined techniques using stent retrievers, aspiration catheters and BGC: the PROTECT ${ }^{\text {PLUS }}$ technique. Clin Neuroradiol 2018; Nov 9. [Epub ahead of print] CrossRef Medline 